Cahiers de recherches médiévales

Journal of medieval studies

$12 \mid 2005$

La tradition épique, du Moyen Âge au XIX ${ }^{\mathrm{e}}$ siècle

\title{
La fortune de Renaut de Montauban
}

\section{Sarah Baudelle-Michels}

\section{OpenEdition}

Journals

Édition électronique

URL : https://journals.openedition.org/crm/2222

DOI : $10.4000 / \mathrm{crm} .2222$

ISSN : 1955-2424

Éditeur

Honoré Champion

Édition imprimée

Date de publication : 30 décembre 2005

Pagination : 103-114

ISSN : 1272-9752

Référence électronique

Sarah Baudelle-Michels, "La fortune de Renaut de Montauban », Cahiers de recherches médiévales [En ligne], 12 | 2005, mis en ligne le 30 décembre 2008, consulté le 15 décembre 2022. URL : http:// journals.openedition.org/crm/2222 ; DOI : https://doi.org/10.4000/crm.2222

Ce document a été généré automatiquement le 15 décembre 2022.

Tous droits réservés 


\title{
La fortune de Renaut de Montauban
}

\author{
Sarah Baudelle-Michels
}

1 Aucun texte épique médiéval n'a bénéficié d'une telle fortune populaire que Renaut de Montauban. Son intertextualité, sa tradition manuscrite, les adaptations en langues étrangères ainsi que la vitalité de ses remaniements incessants attestent le pouvoir de fascination d'un récit qui a connu une longévité exceptionnelle: les quatre frères Aymon - Allard, Renaud, Guichard et Richard -, leur cousin l'enchanteur Maugis et surtout le cheval faé Bayard ont en effet survécu continûment, du Moyen Age à nos jours, dans l'imagination populaire et dans la tradition écrite, sans avoir souffert des siècles d'oubli et de silence qui ont frappé les autres textes épiques tardivement réhabilités au XIX ${ }^{\mathrm{e}}$ siècle.

De l'auteur ou de la date de l'œuvre originelle, on ne sait rien de précis. Un faisceau d'arguments permet toutefois d'en fixer la date aux entours de 1200 : ainsi la chanson, dans un passé carolingien de convention, montre Renaud qui reconquiert Jérusalem, en une revanche fictionnelle et un transfert épique compensatoire ${ }^{1}$.

3 Même dans sa version brève, la chanson est d'une impressionnante envergure, propice aux rebondissements et aux structures itératives. L'argument général en est la faute de Renaud envers Charlemagne, et l'inextinguible courroux que l'empereur en conçoit, la tension dramatique se fondant dès lors sur le caractère apparemment insoluble d'un conflit assez grave pour s'étendre sur plusieurs décennies.

\section{La geste de Montauban}

Dès le XIII ${ }^{e}$ siècle, ce texte fait preuve d'une vitalité extraordinaire, faisant l'objet de nombreuses refontes dont la complexe filiation manuscrite a été minutieusement étudiée par F. Suard ${ }^{2}$ et Ph. Verelst ${ }^{3}$. Le cœur du cycle lui-même, Renaut de Montauban, est sujet à de continuelles transformations. Il en existe une version en vers dite traditionnelle et une remaniée, beaucoup plus longue. Deux des 11 manuscrits de la version traditionnelle ont été édités entièrement, le manuscrit Douce $D^{4}$, qui donne la version la plus ancienne et le manuscrit La Vallière $L^{5}$. Ce sont ces deux manuscrits qui sont donc les plus communément cités lorsque l'on se réfère à Renaut de Montauban. 
Mais la confrontation avec les autres manuscrits que permet l'édition synoptique de quelques passages ${ }^{6}$ montre que le texte, particulièrement mouvant, est sujet à un « constant travail de réfection » allant « dans le sens de l'amplification narrative ${ }^{7}$.

Dès le XIII siècle, on a greffé sur le Renaut des textes "satellites" (Ph. Verelst), en amont pour relater les enfances de Maugis, de son frère Vivien et de Renaud avec Maugis d'Aigremont qui s'apparente à un roman d'aventures,et plus tardivement sa brève suite Vivien de Monbranc. En aval, la tradition critique détache une continuation du manuscrit N (B.N. fr 766) qu'elle intitule La Mort de Maugis. On voit que Maugis est très vite devenu le héros d'un cycle à lui tout seul, comme dans la saga norroise avec la Mágus saga jarls. Ces textes adjoints s'efforcent de répondre à des questions laissées pendantes dans le Renaut, portant notamment sur l'origine de la haine entre les Aymonides et Charlemagne, celle du pouvoir magique de Maugis, ou encore sur Bayard.

6 Au cours du XIV ${ }^{\mathrm{e}}$ siècle, apparaît une version remaniée de Renaut que Ph. Verelst qualifie d'« aristocratique » en raison de son public présumé et de sa préciosité. Il en a édité l'un des deux représentants connus, le manuscrit $R$ (daté d'environ 1440), qui, avec ses plus de 28000 vers, est pourtant vraisemblablement incomplet. Sur un Renaut fortement abrégé - ainsi le prologue sur Beuves d'Aigremont, si long qu'on l'a un temps considéré comme une chanson distincte, est ici réduit à l'adoubement et de la querelle aux échecs-, le narrateur ajoute une matière nouvelle privilégiant épisodes romanesques et courtois, ou aventures surnaturelles, et pratique une constante surenchère: le héros, en particulier, devenu l'aîné des quatre frères est présenté comme un Élu qui ramène les reliques de la Passion. Dans un fragment spécifique au manuscrit $B$ (inspiré de La Mort de Maugis du manuscrit $N$ ), le narrateur relate aussi la mort du magicien Maugis devenu Pape et celle des trois frères survivants, piégés dans une grotte et enfumés par les conseils de Ganelon ${ }^{8}$.

La version primitive et la version remaniée ont été toutes deux mises en prose au cours $\mathrm{du} \mathrm{XV}^{\mathrm{e}}$ siècle. Sans qu'aucun manuscrit en soit l'hypotexte spécifique, elles sont à la source dedeux familles, l'une traditionnelleet l'autre amplifiée. Cette dernière, qui accorde une large place au merveilleux, est représentée par des manuscrits somptueusement enluminés de Renaut ${ }^{9}$ et offrent un texte complet, contrairement au remaniement en vers. Cette "somme épique " ${ }^{10}$, qui s'apparente à un roman généalogique, intègre Maugis d'Aigremont (ce que ne faisait pas $R$ ) et, dans la partie qui conclut le cycle, relate longuement les aventures du petit-fils de Renaud, Mabrien, lequel surclasse largement son aïeul. Cette longue prose restera sans descendance dans les versions imprimées ${ }^{11}$ de Renaut de Montauban, tandis que Maugis et Mabrian seront édités et que Maugis passera même dans la littérature de colportage.

8 La seconde famille regroupe des manuscrits qui adaptent la version courte et traditionnelle de la chanson. Si le passage du vers à la prose n'implique pas de changement diégétiques majeurs, l'abandon du caractère formulaire de l'expression épique entraîne un changement de registre notable: le récit perd en poéticité et devient véritablement prosaïque, le dérimage supprimant toute cette sensibilité à la matérialité du monde sensible, tout l'appareil d'idéalisation, toute l'aura du texte.Ici les personnages gagnent en lisibilité ce qu'ils perdent en grandeur.

9 Le manuscrit Arsenal $3.151(A r)$, le seul à conserver le Prologue ${ }^{12}$ faisant remonter à Beuves l'origine de la révolte, représente le mieux ce qui pouvait être la source de la descendance imprimée d'un texte désormais connu sous le titre des Quatre Fils Aymon ${ }^{13}$. Le premier incunable est presque contemporain de la longue prose puisqu'il date de 
1483-85. Le texte de la prose traditionnelle, désormais mis en chapitre, y est transposé avec une fidélité certaine. Six autres incunables nous sont parvenus. Ceux de Jehan de Vingle, enrichis de bois magnifiques, n'ont pas manqué d'attirer l'attention des imprimeurs de la Renaissance, qui ont (à notre connaissance) donné vingt-trois éditions du texte. Peu à peu, la qualité de ces éditions décline, étant altérée par une mise en page compacte, un papier médiocre, et un nombre grandissant de coquilles. L'illustration elle-même, fréquente, est parfois peu logique et utilise des bois de réemploi. C'est ce corpus de la Renaissance qui servira de vivier aux éditions ultérieures, aucune ne remontant au texte source. L'idée de diffuser largement pour quelques sous des romans de chevalerie tirés de ce fonds ancien apparut au XVII ${ }^{\mathrm{e}}$ siècle, notamment à Troyes avec la famille Oudot puis Garnier. Le titre des Quatre Fils Aymon ne devait plus quitter le catalogue de la Bibliothèque Bleue et l'on dénombre plus d'une centaine de ces livrets de colportage jusqu'au XIX ${ }^{\mathrm{e}}$ siècle $^{14}$. Cette lignée ininterrompue de réécritures se caractérise par une grande inertie éditoriale : si le texte est peu à peu modernisé et surtout abrégé pour des raisons stylistiques et économiques, les éditions «bleues" sont en fait assez fidèles aux premiers incunables et, partant, à la mise en prose traditionnelle de Renaut de Montauban. Car les rééditeurs des Quatre Fils Aymon sont dans l'ensemble des artisans plus que des artistes. Ils ont en vue, humblement, de rendre plus lisible un récit qu'ils affectionnent. Depuis le $\mathrm{XX}^{\mathrm{e}}$ siècle enfin, l'une des réorientations majeures de l'histoire des Quatre Fils Aymon est sa spécialisation dans la littérature destinée à la jeunesse.

Il reste que les réécritures des Quatre Fils Aymon ne sont pas toujours aussi respectueuses de leur modèle, et que quelques remanieurs lettrés, depuis le XVIII siècle, n'ont pas hésité à s'en approprier le texte pour faire œuvre, non pas de transcripteur, mais d'écrivain, exploitant pour ce faire toutes les virtualités du texte originel. L'éclectisme de ces compositions les rend difficiles à présenter ici. Ces rééditions successives nous font mesurer l'étendue des ressources de la geste initiale.

\section{Révolte et esprit de révolte}

Plutôt que d'adopter l'ancienne classification lignagière de Bertrand de Bar-sur-Aube, qui rattachait la chanson de Renaut de Montauban au cycle de Doon de Mayence, on préfère de nos jours la classer thématiquement dans le cycle des barons révoltés, cycle qui dépeint une rébellion d'autant plus séditieuse dans l'univers de la fiction carolingienne qu'elle est soigneusement contrôlée dans la réalité capétienne. C'est que le conflit entre Charlemagne et les Aymonides est l'épine dorsale de l'œuvre. Le terme de "révolté » est toutefois quelque peu inapproprié en ce qui concerne le héros Renaud: si le fils d'Aymon de fait est un rebelle, il n'est pas pour autant animé d'un esprit luciférien de rébellion. Ce que raconte la chanson de geste, ce n'est pas tant l'hybris condamnable de vassaux égarés dans l'insoumission, ce n'est pas l'insurrection délibérée guidée par un (sans doute anachronique) idéal politique de liberté, ni même quelque conflit purement féodal où les factieux seraient animés par leurs intérêts ou leur volonté de puissance, c'est au contraire la démesure entêtée d'un suzerain aveuglé par l'esprit de vengeance. Ainsi, loin de s'obstiner dans une sédition coupable, Renaud, tenaillé par le remords et la hantise que sa révolte ne soit pas juste, ne cesse en réalité de chercher le moyen de regagner la faveur royale; loin de prendre l'initiative des combats, il subit cette guerre comme une calamité et une malédiction. Mais toutes ses 
tentatives pacifiques de conciliation se heurtent pathétiquement à l'inextinguible vindicte impériale. Dans les autres chansons de la révolte, la démesure des rebelles les mène soit à la mort (Raoul, Bernier), soit à la solitude (Ogier). Dans Renaut de Montauban, au contraire, le héros rassemble tous les siens derrière lui et, nimbé de la gloire du martyr, accède à l'immortalité de la sainteté tandis que Charlemagne, abandonné par ses barons sous les remparts de Trémoigne, risque un temps l'infamante solitude sanctionnant l'invalidité de sa politique. En somme, le vassal ne se révolte que parce que la figure impériale se fissure, sans que la chanson sombre pour autant dans le manichéisme.

\section{La crise de la figure royale}

12 L'image de Charlemagne est particulièrement dégradée dans Renaut de Montauban, surtout si on la juge à l'aune des représentations traditionnelles de l'hagiographie historique et de la première génération des textes épiques. De fait, tous les conseillers de l'empereur en viennent à un moment ou un autre à exprimer des réserves sur sa conduite, ne se privant pas de le lui dire en face avec un franc-parler déconcertant ${ }^{15}$. Certes on a l'injure facile dans la tradition épique, mais il est indéniable que Charlemagne est ici loin d'incarner les valeurs qui s'attachent à son rang et à sa renommée. Impulsif, obstiné, crédule et vaniteux, pratiquant à plusieurs occasions l'infamie du parjure, le chef connaît les mêmes pulsions que ses hommes, tout particulièrement ces jeunes têtes brûlées d'ordinaire tant décriées ou ceux du lignage des traîtres. Son tort majeur est d'être incapable de pardonner parce qu'il confond sa fonction et sa personne. Stratège sans génie, il alterne les défaites peu glorieuses et les victoires aussi temporaires qu'inefficaces, tout en manifestant une conception autoritaire et brutale du pouvoir. C'est sur cette faille constitutive du personnage que repose la tension dramatique de Renaut de Montauban, l'homme n'en restant pas moins « Karles de France li forz rois corronnez »(D, v. 6).

13 Car des quatre frères ou de leur ennemi, qui a raison? Lors du duel de type ordalique entre Renaud et Roland, une nuée opportune empêche de départager clairement les adversaires, évitant au narrateur d'avoir à se prononcer. Sans doute les modes de présentation des personnages orientent-ils notre sympathie vers les fils d'Aymon, mais le suzerain n'en reste pas moins le souverain. Et le texte nous le dit à plusieurs reprises : "Qui son segnor guerroie il ne fet pas reson » (D, v. 284). Comme le souligne D. Boutet, « au bout du compte, cette violence opère une sorte de catharsis, et le dernier mot revient toujours à l'ordre royal $\aleph^{16}$. La chanson ne cherche donc pas à mettre en cause le principe de la monarchie, mais s'emploie à explorer toute une casuistique des liens féodo-vassaliques. Avec Beuves, seul rebelle à payer de sa vie sa révolte, on assiste au choc de l'intérêt particulier contre la puissance souveraine; avec les Pairs, qui se désolidarisent peu à peu de leur empereur, à l'antagonisme entre l'intérêt général et le désir personnel de vengeance. Le personnage du duc Aymon, le représentant de la vieille génération unie par le plus fort des liens vassalique, ouvre un débat sur la prééminence des lois temporelles (la soumission au suzerain) sur les lois naturelles (l'amour des siens) ; enfin, avec les quatre fils Aymon, le récit analyse le conflit entre la légitimité individuelle de la révolte et son illégitimité sociale. La fiction explore ainsi les différentes formes de rébellion, de sorte que le problème des pouvoirs respectifs du suzerain et de ses vassaux est posé dans toutes ses dimensions. Et avec le roi Yon, 
«traître occasionnel $\aleph^{17}$ que Renaud refuse pourtant d'abandonner, notre héros prouve bien l'intangibilité de la figure royale. Ainsi, alors même que les sortilèges de Maugis ont livré Charlemagne à Renaud, ce dernier s'agenouille devant son prisonnier resté impavide et martial, le héros refusant comme un tabou de porter la main sur son souverain. On peut d'ailleurs estimer que Charlemagne sort grandi des vicissitudes qu'il aura eu à affronter, le système politique parvenant en outre à résoudre de lui-même la crise qui l'aura ébranlé.

\section{Une subversion circonscrite}

14 À défaut de pouvoir ou vouloir agresser directement l'empereur, les vassaux s'en prennent métonymiquement aux symboles de la royauté en dérobant tour à tour selon une structure itérative, le trésor, l'aigle d'or, l'épée et, à deux reprises, la couronne de Charlemagne. Plus grave, à la faveur d'une plaisante et attentatoire réification, c'est Charlot, le fils de l'empereur qui en vient à être volé, placé dans un sac et chargé comme un ballot sur Bayard. L'aspect subversif de ces actes séditieux est indéniable, voler la couronne pouvant s'apparenter à une destitution symbolique. Mais encore convient-il de marquer que ces rapines sont pour l'essentiel le fait d'un personnage bien spécifique, l'enchanteur Maugis.

Maugis est un personnage capital, moins toutefois au plan de la diégèse qu'en vertu des effets cathartiques produits par la répétition de ses interventions comiques. "Porteétendard du camp de la révolte $»^{18}$, il pousse très loin la rébellion, ce dont Charlemagne a bien conscience puisqu'il fait de sa reddition la condition nécessaire à toute réconciliation. Certes, le «bon larron » est un opposant de taille, étant tout à la fois un valeureux combattant et un enchanteur, mais, somme toute, il ne fait qu'un usage modéré de la magie, qui ne résout jamais à elle seule la moindre crise. En fait, si Maugis pour Charlemagne est la personne à abattre, c'est qu'il s'en prend au pouvoir impérial dans sa trifonctionnalité. Il est évident que la fonction guerrière est constamment mise à mal dans cette chanson où l'on voit cinq barons résister près de deux décennies à l'armée impériale tout entière, l'empêchant par là-même de mener de plus nobles conquêtes. Mais l'enchanteur va plus loin. Dans une scène magistralement étudiée par Alain Labbé, on voit cet « insaisissable Protée de l'invention verbale » ${ }^{19}$ manipuler avec verve Charlemagne en se faisant prendre pour un pèlerin malmené... par Maugis et réclamer l'aide de l'empereur. Celui-ci, crédule, en vient à s'agenouiller pour lui mettre dans la bouche les meilleurs morceaux de nourriture au cours d'un dérisoire simulacre de communion. Ce passage carnavalesque conteste manifestement la puissance royale dans sa fonction nourricière. Charlemagne l'éternel volé, le responsable de l'« ensauvagement » ardennais et de la famine à Montauban, lui qui n'aura livré que de stériles conflits, est ici explicitement tourné en dérision par un retournement du motif traditionnel de la largesse royale. Lors de cette même scène, Charlemagne, l'oint du Seigneur, qui s'empresse de toucher le pèlerin mystificateur dans l'espoir d'être protégé de son aura, voit enfin porter atteinte à sa fonction religieuse. Dans Renaut de Montauban, le roi n'est visité ni par les anges ni par les songes prémonitoires. Il n'est plus marqué par le signe positif de l'altérité. Ce n'est plus lui qui entretient des rapports familiers avec le surnaturel mais Renaud, qui finira en saint martyr. Quant à Maugis, il représente un autre type de surnaturel, celui plus inquiétant de l'imaginaire 
païen, le seul susceptible de déstabiliser un temps l'ordre glacé de la royauté carolingienne.

Ainsi le crime de lèse-majesté est pour l'essentiel pris en charge par un magicien, le recours au merveilleux permettant de dépasser le légitimement concevable dans le cadre juridique et moral du système féodal. L'empereur est malmené par un enchanteur, apparenté à la figure du fou. Qui plus est, cette sédition ne durera qu'un temps. Renonçant à la pratique de la magie, Maugis se fera ermite et Charlemagne retrouvera sa superbe une fois le mage neutralisé : la subversion est circonscrite. Mais derrière Maugis se profile le cheval faé Bayard qui, lui, restera au contraire irréductible. Plus encore que Maugis, Bayard incarne la transgression. Explicitement présenté comme faé, le cheval est dès l'origine doté de qualités exceptionnelles, mais les premiers textes n'évoquent pas encore la merveilleuse capacité de sa croupe à s'étirer pour porter la fratrie tout entière. Nous reviendrons sur ce prodige qui finira par devenir sa principale caractéristique. Mais dans les premières versions du Renaut, il n'est nul besoin de ce trait surnaturel supplémentaire pour deviner l'appartenance du cheval à un substrat pré-chrétien : la concomitance de la contrition pieuse de Renaud et de son adieu à Bayard trahit bien la nature païenne du cheval-fée. Il vit en effet en parfaite symbiose avec la forêt des Ardennes, dont les barons s'accordent à reconnaître la dimension féerique, et où il finira par se réfugier définitivement en franchissant un fleuve qui pourrait correspondre à cette « frontière humide» des anciennes croyances celtiques. En élargissant son étude au cycle de Maugis, F. Dubost a bien montré que les origines merveilleuses de Bayard, fruit de l'accouplement monstrueux d'un dragon et d'un serpent et délivré par Maugis des bouches des Enfers, est un représentant du paganisme : «Baiart (...) est associé par la conscience collective à un ordre ancien et païen qu'il importe d'abolir ». La cruauté de Charlemagne à son égard peut dès lors s'en trouver expliquée puisque «le cheval faé représente aux yeux de l'empereur chrétien les forces surnaturelles et les croyances du monde sauvage que sa mission lui impose précisément d'anéantir $»^{20}$. Ainsi, faute de pouvoir se saisir de l'enchanteur disparu dans son ermitage, l'empereur se contente de son substitut dans la féerie. Et les quatre fils Aymon perdent Bayard en même temps que leur fratrie se disloque, par une séparation qui marque précisément leur soumission définitive. Mais tandis que ses maîtres se soumettent, le destrier, dans un ultime défi, retourne à cette Arduenna silva propre à symboliser l'irréductible autonomie d'un monde encore non défriché, antérieur à l'Etat moderne et à tout ordre politique. Symbole de l'indomptable, Bayard incarne donc la figure de la créature sauvage qui ne saurait se soumettre qu'à un héros hors du commun. Pour avoir longtemps chevauché cette monture païenne, Renaud en paiera le prix d'un pèlerinage en Terre sainte, suivi d'un martyre qui le rendront définitivement à la spiritualité chrétienne.

Si Maugis lui sert de modèle en embrassant la vie érémitique, Renaud ira plus loin que son cousin dans cette voie et il commence bien avant lui le long parcours eschatologique menant à la sainteté. Livré à Charlemagne par Yon, avatar de Judas, il subit un premier calvaire sur la Roche Mabon, dans la plaine de Vaucouleurs au plan cruciforme. Tout à la fois pénitent, pèlerin et croisé en Terre sainte, il renonce au monde en devenant portefaix sur le chantier de l'église de Cologne où il meurt en martyre, consacré par de nombreux miracles. Le Seigneur l'emporte sur le seigneur, le Service de Dieu sur le service vassalique. Et la chanson de geste, dont le théâtre connaît un brusque transfert de Montauban en Rhénanie, peut rejoindre la légende 
hagiographique préexistante de saint Renaud à Cologne ${ }^{21}$. La parenthèse subversive est définitivement refermée.

\section{Un monde illusoire}

19 Exilé traqué, déguisé en jeune baragouinant à peine le français, pris pour un ribaud en Terre sainte puis humble maçon à Cologne, Renaud, depuis sa mort au monde dans la forêt ardennaise, est à la recherche de son identité réelle. Cette quête plus romanesque qu'épique, qui s'exprime dans l'errance géographique, est jalonnée de déchirements et d'incertitudes exacerbées dans un monde illusoire où rien n'est définitivement acquis sinon l'héritage des haines et la malédiction d'une faute irrémissible. Car Renaut de Montauban, malgré ses indéniables et réguliers contrepoints comiques - ils sont pour l'essentiel le fait de la gouaille de Maugis -, est dans l'ensemble une chanson très sombre. Démontrant la labilité des êtres et des choses, elle multiplie désordres et crises, travestissements et trahisons et peint un monde privé de ses repères. Dans cette spirale du renversement, les rois sont agenouillés devant les «larrons » ou juchés à reculons sur des mulets( $D$, v. 8215), les preux chevaliers se déguisent en ribauds, le héros rejette la couronne de Jérusalem pour se faire maçon. Le cours même de la Nature voit son ordre troublé: l'aube s'en oscurci, le soleil va troublant ( $D$, v. 1970), le père renie le fils, le fils met à mal la loi du père. De fait les liens familiaux eux-mêmes semblent un temps se fissurer, la crise de l'autorité paternelle redoublant, sur un mode mineur et symbolique, la révolte contre le roi. En entraînant Aymon du côté de Charlemagne, le récit suggère que ce n'est pas seulement la conscience irréductible de son devoir d'obéissance vassalique qui pétrifie Renaud, mais aussi le sentiment confus d'être au bord du parricide. En définitive heureusement, Aymon se substitue à Charlemagne dans sa fonction nourricière en bombardant de jambons Montauban affamée. La loi de l'amour triomphe, la solidarité familiale aussi.

20 S'il est un thème positif qui résistera à cette peinture critique des valeurs d'allégeance, c'est bien l'idée que la parentèle doit offrir un front commun à l'adversité. Derrière Renaud, tous font bloc, le cousin Maugis, qui a un lourd passif à régler avec Charlemagne, mais aussi la mère miséricordieuse qui rend leur humanité à ses fils ensauvagés par sept années de relégation dans la forêt, le cousin Ogier, qui fait de son mieux pour ménager ses cousins à Vaucouleurs, et bien sûr les trois frères de Renaud.

\section{Renaud et ses frères}

De fait, le noyau stable du récit, celui autour duquel les adaptateurs ont bâti leurs remaniements, est ce thème central de la fraternité exemplaire. De nombreux chercheurs se sont penchés sur ses origines et ses significations possibles. J. Thomas ${ }^{22} \mathrm{y}$ voit un redoublement du couple épique qui associe fortitudo et sapientia :à un héros preux (Renaud, Richard) correspond un héros sage (Allard, Guichard). F. Suard, qui réexamine ce motif du binôme, en souligne la surenchère (Renaud "récapitule les vertus de tous ${ }^{23}$ ) et le dynamisme : les couples ne sont pas figés, d'autant que Maugis s'y adjoint occasionnellement tandis que Renaud finit pas s'en affranchir. Quant à J. Grisward, il remonte au schéma trifonctionnel indo-européen ${ }^{24}$. Quelles que soient les lointaines motivations de la constitution du groupe, les quatre fils Aymon forment une «phalange » (F. Suard), certitude que finira par emblématiser le thème de la monture 
unique, symbole idéal de la solidarité fraternelle. Comme le fera dire plaisamment $\mathrm{H}$. Closson à un des frères dans une réécriture moderne ${ }^{25}$, Bayard est « le cinquième de nous quatre!». De même que le titre des Quatre Fils Aymon l'a emporté sur Renaut de Montauban parce qu'il privilégie la fratrie ${ }^{26}$, de même le motif de l'unique destrier chevauché en même temps par les quatre frères éclipse au fur et à mesure des remaniements les autres traits de Bayard.À l'origine, dans la version $D$, tout au plus Bayard est-il capable, dans les moments critiques, de prendre en croupe un cavalier supplémentaire. Même si des manuscrits ultérieurs introduisent ponctuellement l'image du cheval qui allonge sa croupe pour porter les quatre frères - «Baiars en porte IIII quant il est bien hastés ${ }^{27}$-, cela ne semble être envisagé que comme une virtualité relevant de l'exagération épique. Or, toute la tradition imprimée, depuis les incunables jusqu'aux éditions d'Epinal, reste fidèle au schéma de base : au mieux, on précise que Bayard aurait été capable de les porter tous les quatre. Il faudra attendre des textes des $\mathrm{XVIII}^{\mathrm{e}}$ et surtout $\mathrm{XIX}^{\mathrm{e}}$ siècles pour que le récit intègre explicitement ce substrat légendaire. Pourtant, et M. Piron l'a souligné au sujet du premier incunable ${ }^{28}$, les représentations de Bayard portant les Aymonides au complet abondent dans les versions imprimées. Si l'illustration fait si souvent fi du texte, c'est que l'image des quatre frères sur un même cheval appartient à un fonds folklorique indépendant de tout support écrit.

Depuis la seconde moitié du XIV ${ }^{\mathrm{e}}$ siècle, on trouve cette représentation sur de multiples enseignes $^{29}$, tapisseries ${ }^{30}$ et autres supports. Mais au $\mathrm{XV}^{\mathrm{e}}$ siècle «le motif désormais légendaire va connaître sa pleine consécration $»^{31}$. Le prouve l'une des mises en prose de Renaut de Montauban datée de 1462qui, dans "une curieuse mise en abyme " mentionne ces « figures d'enseignes », et encore «la figure pourtraicte en maint lieu » de ceux qu'on voit « chevauchans et seans tous quatre sur ung cheval armez, comme s'ilz venoient de la guerre $»^{33}$. L'iconographie sociale prouve donc la persistance d'une tradition orale autonome qui finit par pénétrer dans l'écrit. Qu'un matériau mythologique qui avait subsisté dans l'imaginaire collectif indépendamment des ouvrages publiés ait conservé assez de vigueur pour s'imposer aux éditeurs des époques récentes, voilà qui est d'autant plus inattendu que les réécritures imprimées souscrivent aux principes de crédibilité et de logique narrative.

\section{Les grandes tendances des réécritures}

En effet les remaniements vont majoritairement dans le sens d'une rationalisation du récit. Même les versions remaniées du XIV ${ }^{e}$ qui pratiquent volontiers la surenchère merveilleuse s'efforcent de donner une origine rationnelle à ces manifestations (Maugis dans $R$ aurait ainsi appris son art à Tolède). Quant aux mises en prose, elles privilégient les éléments informatifs et narratifs. Dans le corpus des versions imprimées, on observe aussi le plus souvent une réticence à conserver les données féeriques ou même chrétiennes, l'essentiel étant raboté, version après version. Ainsi, la part de plus en plus faible consacrée à l'épisode en Terre sainte montre bien que les nouveaux rédacteurs n'en reprennent pas à leur compte l'orientation eschatologique. Le merveilleux ne semble donc pas un facteur aussi déterminant qu'on a pu le penser pour expliquer la survie de la chanson.

24 L'autre grande tendance va dans le sens d'une réhabilitation de l'empereur. Déjà dans $R$, volontiers moralisateur, c'est Ganelon, «traitre de nature » (Ph. Verelst), qui, pour 
l'essentiel, prend sur lui la face noire de Charlemagne ${ }^{34}$. On retrouve cette même tendance chez les adaptateurs populaires qui, non contents d'atténuer l'esprit de révolte de leurs héros, renforcent à travers les interventions du narrateur la légitimité $\mathrm{du}$ pouvoir monarchique. Si ces remanieurs se sont presque tous efforcés, contrairement à la logique du récit, d'atténuer la perfidie originelle du roi, c'est pour des raisons à la fois esthétiques et culturelles - l'hybris épique et sa démesure cruelle n'étant plus comprises -, mais surtout parce que l'image de Charlemagne avait suffisamment grandi dans les mentalités pour n'être plus compatible avec l'odieux portrait que le Renaut donne du roi des Francs. À partir du XVIII ${ }^{\mathrm{e}}$, les versions dérivées réhabilitent l'empereur en le rendant plus humain tandis que certaines soulignent même la grandeur de cette ire impériale qui s'identifie au pouvoir royal. De fait, rares sont les relectures qui exploitent les virtualités politiques du motif de la sédition : on n'en a trouvé que deux, l'une présentant Renaud comme un anarchiste avant l'heure ${ }^{35}$, l'autre, écrite sous l'Occupation allemande, comme un résistant à l'oppresseur germanique ${ }^{36}$.

Mais le plus frappant est encore ce courant de fond qui soumet irrémédiablement la vieille épopée aux normes d'un classicisme diffus. Deux transformations sont ici manifestes: d'une part l'abandon, au nom d'une certaine bienséance, des outrances chères à l'épopée, d'autre part, la recherche d'une cohérence tonale qui réduise autant que possible l'hétérogénéité des registres, notamment la juxtaposition archaïque du comique et du pathétique caractéristique de la chanson originelle.

L'examen de la fortune posthume inégalée de Renaut de Montauban semble déboucher sur une aporie : si c'est la perfection du texte primitif qui lui a valu son exceptionnelle vitalité éditoriale, comment expliquer que ce même texte ait subi tant de transformations? Pour F. Suard, l'extraordinaire audience du poème au cours des siècles repose sur le fait que le texte est "à la charnière de l'épique et du narratif », son " originalité » étant de dégager des «scènes puissantes", «fortement individualisées $\|^{37}$ et de réussir en même temps à les relier ensemble par le mouvement d'un enchaînement narratif dynamique. Certes, la chanson ne ménage somme toute que peu de pauses lyriques. Mais si une certaine vivacité dramatique la distingue en effet des lourds et monotones appareils épiques des chansons rivales, force est de constater que cette construction dramatique a été altérée par les remaniement successifs, quand bien même n'en était pas modifiées les péripéties majeures. Si la chanson primitive présente donc d'indéniables qualités structurelles, celles-ci ne suffisent cependant pas à rendre compte d'une pérennité qui n'est pas de même nature que celle de l'Iliade dans la mesure où elle intègre, outre le prototype médiéval, toute ses variantes ultérieures. Rendre compte du phénomène éditorial dont a bénéficié l'histoire des Quatre Fils Aymon impose donc de dépasser le constat de la valeur éminente du texte initial.

À cet égard, la thématique jaussienne connue sous le nom d'esthétique de la réception se révèle particulièrement éclairante pour notre propos. Le modèle proposé par Jauss est dialogique : il pense la réception des œuvres comme un jeu de questions et des réponses. Si un texte comme Renaut de Montauban a pu durer si longtemps, c'est par sa capacité à se prêter à de nouvelles relectures. Non pas que chaque génération de lecteurs réinvente l'œuvre. On dira plus justement qu'ils la redécouvrent. Ils ne s'y projettent pas - au sens où ils y mettraient ce qui à l'origine ne s'y trouvait pas -, mais en perçoivent des significations jusqu'alors inaperçues. Ainsi les réécritures ont le 
mérite d'avoir rendu lisibles ou plus explicites des données dont l'essentiel figurait déjà dans leur modèle, comme autant de suggestions à suivre, de pistes qu'il suffisait d'emprunter, de virtualités. Dans cette perspective, les remaniements successifs de Renaut de Montauban forment un tout avec la chanson de geste dont ils sont issus, et cet ensemble constitue d'une certaine manière une même œuvre. Il reste que l'esthétique de la réception, qui permet à bien des égards de rendre compte de la fortune des Quatre Fils Aymon sans retomber dans les ornières d'une définition académique du chefd'œuvre, revient à proposer un modèle herméneutique certes opératoire mais dont la généralité ne rend pas justice aux spécificités du Renaut de Montauban.

Si l'on considère l'article classique où $\mathrm{M}$. Bakhtine oppose épopée et roman ${ }^{38}$, on ne peut qu'être frappé de voir que notre chanson présente, dès sa version la plus ancienne nombre de traits caractéristiques du genre romanesque. Parmi ces phénomènes constitutifs de ce que le formaliste russe appelle la « romanisation» du récit épique c'est-à-dire sa transformation progressive en roman -, il faut relever avant tout l'esprit de relativité qui le détache complètement de l'absolu épique : relativité du pouvoir quand l'autorité royale est contestée par tous, relativité des liens du sang quand le père renie ses enfants, fragilité des frontières entre l'humain et l'inhumain quand un cheval en remontre aux hommes, ou que des hommes vivent comme des bêtes dans la forêt, relativité même de l'absolu théologique quand des créatures païennes menacent et ridiculisent le chef temporel de la Chrétienté. Le plus frappant comparé à la topique ordinaire de l'univers épique, ne réside-t-il pas dans les hésitations, les remords, les moments de désarroi du héros, qui n'a plus rien d'un seigneur de la guerre sinon la vaillance, mais qui préfigure une conscience moderne par son abandon des certitudes transmises et des valeurs absolues? La dynamique même du récit, avec ses louvoiements dans un univers où rien n'est définitivement acquis ne doit-elle pas sa force et sa raison d'être, moins à un art habile du suspense qu'à une vision du monde déjà déprise des rigidités de l'absolu épique ? Cette histoire qui semble réticente à trancher la question du bien et du mal et laisse finalement ouverts plusieurs enjeux majeurs du récit, n'est-elle pas toujours éloignée du monologisme épique? Si l'on admet cette hypothèse d'une chanson de geste déjà gagnée par le romanesque - au sens fort de vision du monde fondée sur la relativité -, bien des singularités du texte initial s'éclairent: son inachèvement sémantique, les scènes de démystification de l'autorité, le dialogisme - quand les points de vue s'opposent au sein d'une même famille ou au cœur irrésolu des personnages, l'émergence de jugements individuels qui subvertissent le système axiologique officiel - et encore des traits plus subtils décrits par Bakhtine, la dissociation entre "l'être véritable » et "l'aspect extérieur » du héros, le sentiment d'une inadéquation à son destin (n'ayant pas de haine, il n'était pas fait pour la révolte), la polyphonie (par les changements de registre) et le délitement même des bornes géographiques qui au seuil des Ardennes, produit cette ouverture vers l'infini spatial typique de la chronotopie romanesque. Autant d'indices d'une romanisation précoce de ce récit épique, comme si le génie des premiers jongleurs avait perçu que le thème fictionnel de la subversion politique ne pouvait s'accorder aux rigidités de l'épos, et que la mise à l'épreuve de l'absolu royal entraînait nécessairement la contestation parallèle des normes stylistiques de la chanson de geste et de l'intangibilité de l'absolu épique. 


\section{NOTES}

1. Jérusalem avait été reprise par Saladin en 1187. Renaud semble être également une projection de Richard Cœur-de-Lion, qui libéra Acre lors de la troisième croisade, en 1191.

2. "Le développement de la Geste de Montauban en France jusqu'à la fin du moyen âge ", Romance epic. Essays on a medieval literary genre, H.-E. Keller, Kalamazoo, 1987, p. 141-161.

3. "Le cycle de Renaut de Montauban: aperçu général et réflexions sur sa constitution", Cyclification, The Development of Narative Cycles in the Chanson de Geste and the Arthurian Romances, B. Besamusca, W. P. Gerritsen et alii, Amsterdam, 1994, p. 165-170.

4. J. Thomas, "Renaut de Montauban », édition critique du manuscrit Douce, Genève, Droz, 1989.

5. F. Castets, "La Chanson des Quatre fils Aymon", d'après le manuscrit La Vallière, Montpellier, Coulet, 1909. Selon J. Thomas, ce manuscrit ne mérite nullement la place éminente qui lui a été accordée.

6. L'Épisode ardennais de "Renaut de Montauban", édition synoptique des versions rimées, Bruges, De Tempel, 1962 ; A. Negri, L'Episodio di Vaucouleurs nelle redazioni in versi del «Renaut de Montauban », Patron, Bologna, 1996.

7. F. Suard, op. cit., p. 146.

8. Il s'agit d'un manuscrit hybride avec deux fragments en vers et le reste en prose. La partie en prose correspond à la partie en vers traditionnelle. Cf. Ph. Verelst: «Renaut de Montauban. Deuxième fragment rimé du ms. de Londres, B.L.. Royal 16 G II (« B »).Edition critique », Romanica Gandensia, 21, Gand, 1988.

9. Celui exécuté en 1462 pour Philippe le Bon et enluminé par Loyset Liédet, Ars. 5072-75 ( $\mathrm{Am}$ )et pour le cinquième volume Munich, Bayerische Staatsbibliothek, Gall. 7, et celui de Pommersfelden, Schönborn 311-2 (Pm).

10. F. Suard, op. cit, p. 152.

11. À l'exception de l'adaptation pour la «Bibliothèque universelle des romans » parue en juillet 1778.

12. Avec Sloane 960 (Sl) qui découle d'une version ancienne proche de D.Ce prologue se retrouve dans toute la descendance imprimée.

13. Dans le manuscrit $M$ (Montpellier, H 247), vraisemblablement de la seconde moitié du XIV e, la chanson est déjà annoncée comme le rommans dez . iiij. fix Aymon.

14. Ils sont imprimés à Troyes, Paris, Rouen, Limoges, Avignon, Carpentras, Lille, Montbéliard ou Épinal. Pour une liste détaillée, cf. S. Baudelle-Michels, De « Renaut de Montauban » aux « Quatre Fils Aymon ", les avatars d'une chanson de geste, Champion, 2005 [à paraître].

15. Ainsi, lorsque leur souverain cherche un volontaire pour pendre Richard tombé entre ses mains, Turpin, Roland ou encore Olivier, dans des laisses parallèles (D, 184 à 191), qualifient son chantage affectif sans ambages : " grant outrage avez dit » (D, v. 9304), « or avez vos mespris » (D, v.9322), «Vos dites grant outrage » ( $D$, v.9352). Ogier en viendra même à regretter que Renaud n'ait pas décapité Charlemagne après l'avoir fait prisonnier « maudite soit or l'eure de Deu et de sa mere,/que Renaus ne vos fist le cief de cest but rere !» (L, vv. 13092-93).

16. Charlemagne et Arthur ou le roi imaginaire, Paris, Champion, 1992, p. 163.

17. Ph. Verelst, "Traître de nature et traître occasionnel : le cas éclairant du Renaut de Montauban », Actes du XI Congrès international de la Société Rencesvals, Barcelone, 1990, t. II, p. 331346.

18. A. Labbé, «Enchantement et subversion dans Girart de Roussillon et Renaut de Montauban ", Chant et enchantement au Moyen Age, Toulouse, Éditions Universitaires du Sud,1997, p. 149. 
19. B. Guidot, "Travestissements, mutations et métamorphoses dans Renaut de Montauban", Echoes of the Epic. Studies in Honor of Gerard J. Brault, D. P. Schenck et M. J. Schenck, Birmingham (Alabama), Summa Publications, 1998, p. 83.

20. Aspects fantastiques de la littérature narrative médiévale (XII ${ }^{e}$-XIII ${ }^{e}$ siècles). L'Autre, l'Ailleurs, l'Autrefois, Paris, Champion, 1991, p. 451.

21. Cf. P. Fiebig, St Reinoldus in Kult, Liturgie und Kunst, Dortmund, 1956.

22. «Les Quatre Fils Aymon. Structure du groupe et origine du thème », Romanica Gandensia, 18, "Études sur Renaut de Montauban », Gand, 1981, p. 47-72.

23. «Ogier le Danois et Renaut de Montauban », Essor et fortune de la chanson de geste dans l'Europe et l'Orient latin, Modène, Mucchi editore, 1984, t. I, p. 202.

24. "Aymonides et Pandava: l'idéologie des trois fonctions dans Les quatre fils Aymon et le Mahabharata ", Essor et fortune de la chanson de geste, op. cit., p. 77-85. Pour J. Griswald, Aalard, le frère avisé, représente la première fonction juridique, Renaud et sa morphologie de géant la fonction guerrière, tandis que Guichard et Richard incarnent la troisième fonction associée à l'opulence.

25. Le Jeu des Quatre fils Aymon, Bruxelles, éd. Durendal, [1942], p. 149.

26. Cf. S. Baudelle-Michels, «Pourquoi Renaut de Montauban a survécu sous le titre des Quatre Fils Aymon ?», Ateliers,22, "Topiques romanesques : réécriture des romans médiévaux (XVI ${ }^{\mathrm{e}}-\mathrm{XVIII}{ }^{\mathrm{e}}$ s.) ", É. Gaucher et F. Lestringant, 1999, p. 35-42.

27. $L$, v. 1546. Cf. également $P$, v. 584, et $V$, v. 2438 .

28. M. Piron, « Le cheval Bayard, monture des Quatre Fils Aymon, et son origine dans la tradition manuscrite », Romanica Gandensia, 18, op. cit., p. 153-170.

29. À Anvers par exemple, au XVII ${ }^{\mathrm{e}}$ siècle, il y avait neuf ou dix maisons portant l'enseigne «Aux Quatre Fils Aymon" ou «Au cheval Bayard». Cf. C. P. Serrure et A. Voisin, Livre de Baudoyn, Bruxelles, 1836, p. 25.

30. Cf. E. Müntz, «La légende de Charlemagne dans l'art du Moyen Age », Romania, 14, 1885, p. 321-342.

31. M. Piron, « Le cheval Bayard... », op. cit., p. 159.

32. Ph. Verelst, "Texte et iconographie: une curieuse mise en abyme dans un Renaut de Montauban inédit (XV siècle) », Romanica Gandesnia, 17, 1980, p. 147-162.

33. B.N., fr. 19174 , ff. $20^{\mathrm{v}}-21^{\mathrm{r}}$ et B.N., fr. 19173 , fol. $393^{\mathrm{v}}$.

34. Il ourdit ainsi la course des chevaux ou organise le siège de Montauban. Quant au traquenard de Vaucouleurs, Yon en est le seul responsable.

35. Les Quatre Fils Aymon, Le Livre pour tous, Paris, Cornély, [1899].

36. Herman Closson, Le Jeu des Quatre Fils Aymon, op. cit.

37. «Le développement... ", op. cit., p. 143.

38. "Récit épique et roman» (1941), Esthétique et théorie du roman, Paris, Gallimard, 1996, p. 439-473.

\section{AUTEUR}

\section{SARAH BAUDELLE-MICHELS}

Université de Lille 